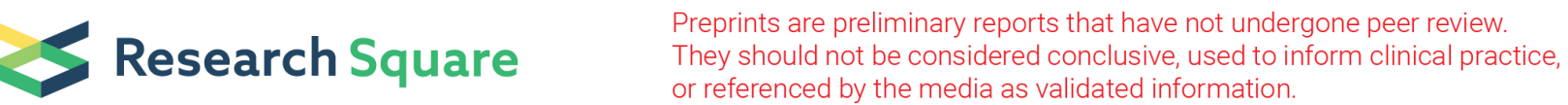

\section{Self-management for people with epilepsy and a history of negative health events (SMART) targeting rural and underserved people with epilepsy: A methodologic report}

\section{Gena Ghearing ( $\sim$ gena-ghearing@uiowa.edu )}

Carver College of Medicine and University of lowa Health Center https://orcid.org/0000-0002-44772868

\section{Farren Briggs}

Case Western Reserve University School of Medicine

\section{Kristin Cassidy}

Case Western Reserve University School of Medicine

\section{Michael Privitera}

University of Cincinnati College of Medicine

\section{Carol Blixen}

Case Western Reserve University Department of Neurology: University Hospitals Neurological Institute

\section{Martha Sajatovic}

Case Western Reserve University School of Medicine

\section{Study protocol}

Keywords: epilepsy, seizure, self-management, rural, hospitalizations, emergency room

Posted Date: April 8th, 2021

DOI: https://doi.org/10.21203/rs.3.rs-401711/v1

License: (c) (i) This work is licensed under a Creative Commons Attribution 4.0 International License. Read Full License 


\section{Abstract}

Background: Many people living with epilepsy (PLWE) reside in rural communities, and epilepsy selfmanagement may help address some of the gaps in epilepsy care for these communities. A prior randomized control trial of a remotely delivered, web-based group format 12-week self-management program (SMART) conducted in Northeast Ohio, a primarily urban and suburban community, demonstrated improved outcomes in negative health events such as depression symptoms and quality of life. However, there is a paucity of research addressing the needs of PLWE in rural settings.

Methods: The present study leverages collaboration between investigators from 2 mid-western U.S. states (Ohio and lowa) to replicate testing of the SMART intervention and prioritize delivery to PLWE in rural and semi-rural communities. In Phase 1, investigators will refine the SMART program using input from community stakeholder. A community advisory board will then be convened to help identify barriers to trial implementation and strategies to overcome barriers. In Phase 2, the investigators will conduct a 6month prospective randomized control trial of the SMART program compared to 6-month wait-list controls, with the primary outcome being changes in negative health events defined as seizure, self-harm attempt, emergency department visit, or hospitalization. Additional outcomes of interest include quality of life and physical and mental health functioning. The study will also assess process measures of program adopters and system end-users to inform future outreach, education, and self-management strategies for PLWE.

Discussion The method of this study employs lived experience of PLWE and those who provide care for PLWE in rural and underserved populations to refine a remotely delivered web-based self-management program, to improve recruitment and retention, and to deliver the intervention. Pragmatic outcomes important to PLWE, payers, and policymakers, will be assessed. This study will provide valuable insights on implementing future successful self-management programs.

Trial registration: The study has been registered at ClinicalTrials.gov (NCT04705441) on January 12, 2021.

\section{Background}

It is estimated that one in 26 Americans will develop epilepsy which remains associated with a high burden of medical complications, decreased quality of life, and premature mortality [1]. A growing body of research supports self-management approaches that can improve outcomes in people living with epilepsy (PLWE). However, because of diverse needs across various sub-populations and known health disparities, it is important to understand how evidence-based epilepsy self-management approaches might impact outcomes across a broad range of PLWE, including those living in rural and semi-rural communities.

While the literature on disparities in epilepsy is growing, comprehensive, comparative data remain limited [2], and most of the literature has not focused on the needs of rural communities. Twenty percent of the 
United States population reside in rural communities, which tend to have a higher rate of poverty, depression, and suicide than urban communities $[3,4]$. There has been a widening gap in healthcare outcomes referred to as the rural mortality penalty, resulting in over 134 excess deaths per 100,000 [5]. A survey of Midwestern neurologists identified low income, lack of insurance, transportation difficulties, stigma, and misperceptions about epilepsy as barriers interfering with epilepsy care in rural regions [6]. Residents of rural communities and other underserved communities may experience health disparities and have multiple areas of unmet need in epilepsy care. A recent targeted review on the social determinants of health in epilepsy recommended engaging patients, especially those from disadvantaged backgrounds, in self-management in order to educate and empower patients [7]. The Managing Epilepsy Well (MEW) Network has been a national leader in developing, testing, and disseminating evidence-based epilepsy self-management programs [8]. One of these, The Self-management for people with epilepsy and a history of negative health events (SMART) program, developed by researchers at Case Western Reserve University (CWRU), is an online 12-week behavioral group-format program targeted to reducing barriers and maximizing facilitators to self-care in high-risk people with epilepsy [9]. SMART has been demonstrated to reduce epilepsy-related complications and improve quality of life [10]. SMART is delivered by Peer Educators (patients with epilepsy) and Nurse Educators. Thus, SMART combines the portability and low cost of a web-based intervention with the personally salient components of behavior modeling that can be obtained by interacting with individuals who have "walked the walk" in living with and coping with epilepsy.

A new SMART 2.0 research study is intended to 1.) Replicate efficacy findings of the original SMART randomized control trial (RCT) in an alternative setting (rural and semi-rural communities), 2.) Incorporate best practices and recommended strategies for enhancing study recruitment and retention, and 3.) Assess program adopter's perceptions of the intervention to inform future implementation and dissemination. This report describes the methods of the planned replication study using a prospective 2site, 6-month RCT design to assess SMART vs. 6-month wait list (WL) control. The study will assess effects on epilepsy complications and other outcomes in a diverse sample with epilepsy including rural people with epilepsy. We hypothesize that at 6-month follow-up, SMART will be associated with reduced negative health events (NHEs) compared to WL. We also expect that SMART will be associated with improved quality of life, functioning, and physical and mental health.

\section{Methods}

This 2-site, 2-phase study (Fig. 1) will investigate the effects of the SMART program on health outcomes in people with epilepsy who have had recent NHEs defined as a seizure, self-harm attempt, emergency department visit, or hospitalization within the last 6-months. The study will be conducted in Ohio and in lowa, led by a team of researchers from CWRU in Cleveland and from University of lowa (UI) in lowa City in collaboration with stakeholders in epilepsy and rural healthcare.

\section{Phase 1}


Phase 1 is intended to set the stage for a successful RCT that will inform future dissemination and implementation efforts should RCT findings be positive. Qualitative methods will be used to collect information from informed and committed local stakeholders. Two focus groups composed of PLWE and their family members from rural communities in lowa and two focus groups composed of rural health providers, social services agency administrators/staff or other professionals working in rural health will be convened to collect information on perceived barriers and facilitators to participation in an epilepsy self-management program, as well as impressions of the SMART self-management curriculum. Information obtained from these local stakeholders will also help the investigators to develop a robust recruitment strategy and a set of practices that will maximize engagement and retention of subjects. Focus groups will be conducted via Zoom technology, last 60-90 minutes, and use a semi-structured interview guide adapted from previous studies in self-management of neurological conditions conducted by the CWRU investigators $[11,12]$. The total number of individuals in the focus groups will be in the range of 32-40 individuals, which is within the recommended sample size range for qualitative studies $[13,14]$.

\section{Community Advisory Board (CAB)}

Building upon strong existing partnerships between members of the study team and epilepsy/neurological care partners across the states of lowa and Ohio, the study team will obtain input from relevant community stakeholders to develop an a priori recruitment and retention strategy intended to maximize enrollment of typically hard-to-reach individuals with epilepsy from underserved and rural communities. The CAB will be composed of up to 16 individuals, including individuals with epilepsy, epilepsy specialists, clinicians and administrators who practice in rural or under-served communities, and representatives from the Epilepsy Association/Epilepsy Foundation.

There will be 3 video-conference calls prior to the RCT. In the first call, CAB members will review the SMART curriculum and identify potential barriers to recruitment and retention. In the second meeting, CAB members will review the list of barriers and suggest solutions and strategies for reaching people with epilepsy who may not be initially help-seeking and discuss potential methods of retaining these individuals in the program once they are recruited. In the third CAB meeting, the list of strategies will be finalized, and the investigators will obtain input on how these strategies might be best operationalized.

\section{Phase 2}

Phase 2 is a prospective 6-month comparative trial of SMART vs. WL in 160 individuals with epilepsy and a recent NHE. Participants will be randomly assigned (1:1 basis) to receive either SMART $(N=80)$ or WL $(\mathrm{N}=80)$ will be randomized using a computer-generated random number generator during the enrollment process. The study staff enrolling the subject will then allocate the treatment or waitlist group in REDCap. The investigators will conduct 8-10 separate and sequential SMART groups with up to 10 individuals in each group. SMART will consist of two main components: a 12-week remotely accessed "intensive" group format stage and a 12-week remotely accessed web/telephone follow-up stage.

\section{Setting and Recruitment}


To assess the potential of SMART to promote behavior change in people with epilepsy and NHEs, the investigators will build on their strong track-record of working with local federal, state, and county entities that provide service to rural and under-served communities. Since SMART is an adjunct to ongoing neurological and medical care, it is expected that study participants will continue to receive services with their regular medical clinicians and providers at all sites.

Participants will have a self-reported diagnosis of epilepsy, be at least 18 years of age, have experienced an NHE within the last 6 months, and be able to provide written informed consent and participate in study procedures. Study entry criteria are purposely broad in order to best represent "real world" people with epilepsy [15]. Actively suicidal/homicidal individuals and those with dementia will be excluded, as will pregnant women who are likely to need different and more intensive treatments.

The study team will attempt to oversample PLWE who live in rural or semi-rural settings. Classification of rurality will be determined based upon the 2013 Rural-Urban Continuum Codes [16] a classification scheme that distinguishes metropolitan counties by the population size of their metro area, and nonmetropolitan counties by degree of urbanization and adjacency to a metro area. The official Office of Management and Budget metro and non-metro categories have been subdivided into 3 metro and 6 nonmetro categories. Each county in the United States is assigned 1 of the 9 codes.

In addition to the RCT participants, the investigators will enroll and train up to 8 Peer Educators using procedures like those conducted in previous work [17]. A Peer Educator will be an individual with epilepsy who has had at least 3 previous life-time NHEs. Informed consent will be obtained for all focus group members, peer educators and RCT participants, by study staff during the enrollment process. Additional consent must be obtained to include the deidentified subject data in the MEW network database. All work will be conducted consistent with Institutional Review Board (IRB) approval at the relevant sites. Adverse events will be reported to the IRB. Regular meetings between the enrolling sites will communicate any changes in protocol. Annual updates will be provided to the CDC, including any adverse events. This is a low risk study, but if a participant is identified as suicidal or homicidal during the screening process or at any time during the study, the study staff interacting with the individual will use existing clinical infrastructure to insure safety and appropriate clinical follow up.

Upon project completion, the investigators will present findings to the UI Prevention Research Center, our project-specific CAB, the CDC MEW Network Coordinating and Collaborating Centers and to community health partners. Findings will be presented at relevant scientific venues and in peer-reviewed publications and may help to scale- up epilepsy self-management program opportunities.

\section{Experimental Intervention}

The SMART curriculum is divided into 2 steps. Step 1 consists of eight group-format 45-60-minute sessions with up to 10 participants per group, which will be collaboratively delivered by a Nurse Educator and a Peer Educator. The intervention will be delivered in a web-based format emphasizing interactive discussion with a secure videoconferencing system. Participants will be able to log on and interact via 
audio and/or video. Telephone call-in will be available for those who do not have internet access. Patients will receive e-mail and phone call reminders of sessions, and make-up sessions will be available. The initial group-session portion of SMART will be completed over 10-12 weeks (Table 1). Following the Step 1 group sessions, Step 2 consists of 3 brief (no more than 15 minutes) monthly web-based or telephone maintenance sessions conducted by the Nurse Educator. Telephone sessions will address on-going issues of epilepsy self-management including treatment adherence. Additionally, the nurse will serve as a facilitator of linkage between the individual's epilepsy care clinicians by providing SMART program status updates to providers.

\section{Table 1 SMART curriculum: Topics covered in each session.}

\begin{tabular}{|c|c|}
\hline Session 1 & $\begin{array}{l}\text { Orientation and introductions; Emphasize ground rules; Establishment of a therapeutic relationship; Facts and myths about } \\
\text { epilepsy and general epilepsy management principles }\end{array}$ \\
\hline Session 2 & $\begin{array}{l}\text { Relationship of epilepsy and stress; Stigma and "double stigma"; Strategies to cope with stigma; Introduction to personal goal- } \\
\text { setting }\end{array}$ \\
\hline Session 3 & $\begin{array}{l}\text { Treatments for epilepsy; Complications of epilepsy; Minimizing epilepsy complications; The importance of daily routine and good } \\
\text { sleep habits }\end{array}$ \\
\hline Session 4 & $\begin{array}{l}\text { Problem-solving skills and the IDEA approach (Identify the problem, Define possible solutions, Evaluate the solutions, Act on the } \\
\text { best solution); Talking with your health care providers; Role play of communication with care providers }\end{array}$ \\
\hline Session 5 & $\begin{array}{l}\text { Nutrition for best physical and emotional health; Substance abuse and its effects on epilepsy; Specific stress-management } \\
\text { approaches }\end{array}$ \\
\hline Session 6 & $\begin{array}{l}\text { Effects of exercise and being outdoors on physical and emotional health; Medication routines; Prioritizing medication side effects } \\
\text { and discussing it with your clinician }\end{array}$ \\
\hline Session 7 & $\begin{array}{l}\text { Social supports and using your available supports; Advocacy groups for epilepsy; A personal care plan to take care of the mind and } \\
\text { the body }\end{array}$ \\
\hline Session 8 & $\begin{array}{l}\text { Normalizing your life in spite of having a chronic but unpredictable condition; Self- management as a life-style; Acknowledgement } \\
\text { of group progress; Setting the stage for Ongoing Illness Management and Recovery (Step 2) }\end{array}$ \\
\hline
\end{tabular}

\section{Waitlist (WL) Control}

Individuals randomized to WL will continue in their usual care. After they complete their 13-week and 6month assessments, which are identical to the SMART group assessments, WL individuals will begin participating in the SMART intervention for the next 12 weeks. Once the WL individuals begin participating in SMART, they will have research assessments at 13 weeks, 6 months, and 12 months after beginning SMART.

\section{Feasibility and Fidelity}

Attendance for each SMART session will be recorded. Acceptability will be assessed at the end of each 12-session group series with a brief self-rated survey. Following Fraser [18], fidelity to the SMART intervention will be assessed quantitatively and qualitatively. Non-interventionist research staff will monitor and assess at least $25 \%$ of group sessions, with each fidelity dimension being rated on a $1-10$ scale. 


\section{Outcomes and Analysis}

Table 2 outlines study procedures. In addition to demographic and clinical information (age, gender, ethnicity, self-reported cumulative medical illness [19, 20]), health literacy will be assessed [21] to detect persons with limited or marginal health literacy. For both the SMART and the WL groups, research assessments will be done at screening (to establish study eligibility), at baseline, and at 13 weeks, 6 months, 12 months, and 18 months after randomization. Members of the waitlist will also be assessed 13 weeks after starting the SMART intervention. All assessments are suitable for remote administration either by phone or using REDCap (Research Electronic Data Capture), a secure web application for building and managing on-line surveys and databases [22]. REDCap will be used for secure data entry and storage. 
Phase 2/RCT procedure - Line 221

\begin{tabular}{|c|c|c|c|c|c|c|c|c|}
\hline PROCEDURES & Screen & Baseline & $\begin{array}{l}\text { Wk } \\
1- \\
12\end{array}$ & $\begin{array}{l}\text { Wk } \\
13\end{array}$ & $\begin{array}{l}\text { Wk } \\
13- \\
\text { Mth } \\
6 \\
6\end{array}$ & $\begin{array}{l}\text { Mth } \\
6\end{array}$ & $\begin{array}{l}\text { Mth } \\
12\end{array}$ & $\begin{array}{l}\text { Mth } \\
18 / \\
\text { EOS }\end{array}$ \\
\hline $\begin{array}{l}\text { Identification of participants: } \\
\text { Inclusion/Exclusion criteria review }\end{array}$ & $\mathrm{x}$ & & & & & & & \\
\hline Informed Consent & $\mathrm{X}$ & & & & & & & \\
\hline Demographics; Health literacy & $\mathrm{X}$ & & & & & & & \\
\hline $\begin{array}{l}\text { Medical History: Cumulative Medical } \\
\text { Burden }\end{array}$ & $\mathrm{X}$ & & & & & & & \\
\hline Randomization & & $\mathrm{x}$ & & & & & & \\
\hline Primary Outcome: Number of NHEs & & $\mathrm{x}$ & & $\mathrm{X}$ & & $\mathrm{X}$ & $\mathrm{x}$ & $\mathrm{X}$ \\
\hline $\begin{array}{l}\text { Secondary Outcomes: Depression: } \\
\text { PHQ-9 Functional status: SF-36 } \\
\text { Epilepsy Control: Seizure frequency }\end{array}$ & & $x$ & & $x$ & & $x$ & $x$ & $x$ \\
\hline Quality of Life: QOLIE-31 & & & & & & & & \\
\hline $\begin{array}{l}\text { Patient factors affecting self- } \\
\text { management: }\end{array}$ & $\mathrm{x}$ & & $\mathrm{x}$ & & & $\mathrm{x}$ & & $\mathrm{x}$ \\
\hline $\begin{array}{l}\text { Epilepsy self-management } \\
\text { competency: ESMS Epilepsy self- } \\
\text { efficacy: ESES Social Support: } \\
\text { MSPSS Epilepsy stigma: SSE }\end{array}$ & & & & & & & & \\
\hline $\begin{array}{l}\text { Program adopter/end-use process } \\
\text { measures: }\end{array}$ & & & & & & & & \\
\hline Patient perceptions & & & & & & & & \\
\hline $\begin{array}{l}\text { Clinician and community partner } \\
\text { referral patterns }\end{array}$ & & & & & & & & \\
\hline SMART Groups attendance & & & $\mathrm{x}$ & & & & & \\
\hline SMART Follow-up phone calls & & & & $\rightarrow$ & & & & \\
\hline SMART Acceptability survey & & & $\mathrm{x}$ & & & $\mathrm{x}$ & & \\
\hline
\end{tabular}

\footnotetext{
Wk = week, Mth = month, NHE = negative health events EOS $=$ end of study
} 


\section{PROCEDURES}

Screen

Baseline

Wk

12

Wk
13

$\begin{array}{ll}\text { Wk } & \text { Mth } \\ 13- & 6 \\ \text { Mth } & \\ 6 & \end{array}$

Mth

Mth

$1218 /$

\section{Wait-list (WL) begins SMART}

(WL outcomes assessed at baseline, 13 weeks, 6 months, 12 months, and

18 months after randomization; Also assessed 13 weeks after starting SMART intervention)

Wk = week, Mth = month, NHE = negative health events

EOS $=$ end of study

\section{Primary outcome:}

The primary outcome will be proportion of individuals with reduction in their total number of NHEs in the prior 6 months at baseline compared to week 24 of the trial. The second primary outcome will be the change in NHE counts in the 6 months prior to baseline compared to week 24 of the trial. NHEs are defined as seizures, emergency department visits, hospitalizations, and self-harm attempts. NHE reporting will be via self-report with corroboration by EHR documentation and report of family/support system informants whenever possible. In addition to total NHEs, we will assess NHEs in each category, including seizure frequency, and length of stay in days for hospitalization NHEs.

\section{Secondary/additional outcomes:}

Additional assessments will include evaluation of depressive symptoms using the PHQ-9, a widely used and validated self-rated depression scale [23]. Functional health status will be assessed using the 36-Item Short Form Survey (SF-36), a multi-purpose, short-form health survey that yields two psychometricallybased components: a physical component summary and mental component summary, which has proven useful for comparing the relative burden of diseases [24]. Epilepsy control will be assessed via selfreported seizure frequency. Quality of life will be assessed with the Quality of Life in Epilepsy (QOLIE-31) self-administered questionnaire which comprises 7 components including seizure worry, overall quality of life, emotional well-being, energy-fatigue, cognitive functioning, medication effect, and social function [25, 26].

Other factors assessed in the trial include epilepsy self-management competency, self-efficacy, social support, and stigma. Self-Efficacy will be measured with an Epilepsy Self-Efficacy Scale (ESES) 2000 version. The ESES was developed based on Bandura's conceptualization of self-efficacy to assess a person's confidence in his or her ability to use self-management strategies. Previous studies show acceptable construct validity and high test-retest reliability in the ESES [27-29]. Social support will be 
measured with the Multidimensional Scale of Perceived Social Support (MSPSS), a 12-item scale that measures an individual's perception of social support provided by family and friends, as well as satisfaction with that support [30]. Stigma for epilepsy will be measured using the 24-item Stigma Scale of Epilepsy (SSE) developed by the Demonstration Project on Epilepsy as part of the WHO/ILAE/IBE Global Campaign against Epilepsy [31,32]. The SSE is a validated, versatile, and sensitive instrument which has been used mainly in resource-poor settings to study stigma in epilepsy.

\section{Process Measures}

Curran and colleagues define process evaluation as a rigorous assessment approach designed to identify potential and actual influences on the conduct and quality of future implementation and dissemination [33]. The process evaluation will target 2 main areas: 1) Identify and describe program adopter's perception of the SMART program, and 2.) "Reach" among clinicians and community partners for SMART referral. Patient perceptions will be assessed via a set of open-ended questions regarding their satisfaction with the program, comprising Likert-style questions assessing positive/negative/neutral components of the program and whether they would recommend participation in SMART to a family member or friend with epilepsy. Referral sources (clinicians, community partners, and other referral sources) will be tracked for all study screens as well as successful enrollments.

\section{Data Analysis}

Descriptive analyses will compare the individuals assigned to SMART or WL using Student's T-test, Fisher's exact test, and non-parametric tests when appropriate. The primary outcome is whether there is a difference in the proportion of individuals with reductions in the total number of NHEs in the prior 6 months, at baseline, and at follow-up ( 6 months after randomization) between SMART and WL, compared using a Fisher's exact test. We will also test for the difference in NHE counts between baseline and followup using a non-parametric Wilcoxon-Mann-Whitney test (the distribution of this measure is expected to be non-normal based on our prior experience). Exploratory longitudinal mixed models with a subject-level random effect will be conducted to investigate a binary variable of no NHEs versus at least one NHE and for a count measure of total NHEs from baseline to 10 and 24 weeks. Explanatory variables will include study site, age, gender, race/ethnicity, number of seizure medications, rural/urban continuum status, and attendance or deviations from study protocol.

The 12-month and 18-month measurements of NHEs will be compared to baseline and 6-month measurements as well. We will also examine change-over-time in the secondary outcomes using parametric and/or non-parametric tests as deemed appropriate for each measure. NHE measurements will reflect the preceding 6-month time period, except for the 13-week time period. The 13-week NHE measurement will reflect the time period following baseline (when individuals participating in SMART will be having their "intensive" group sessions), and it will be used to assess for within-subject differences from baseline to 13-week and 13-week to 6-month time periods. Note that the 13-week to 6-month NHE count will be derived by subtracting the measurement taken at 13-weeks from the one taken at 6-months. This will allow for a greater understanding of the time course for when the expected reductions occur in 
the SMART group, which we expect to mostly occur after the "intensive" 8 group sessions have ended. A secondary analysis of NHEs will include comparison of hospital and emergency department visits. A 2sided Type I error rate of $5 \%$ will be considered significant.

\section{Sample Size Calculations}

Psychosocial interventions can reduce seizures and emergency department visits in people with poorly controlled epilepsy [34,35]. Oosterhuis noted a $50 \%$ reduction in seizures with group psychoeducation [36]. In our original SMART efficacy trial, total number of past 6-month NHEs were reduced by $>50 \%$. Projected sample size is 160 , with 80 subjects per arm. While the web and phone-based access format of SMART optimized study retention and attrition rate in the original RCT was $14.2 \%$, we conservatively assume $20 \%$ attrition at 6 months $(\mathrm{N}=128)$. A 2-sided, 2-sample, Fisher's exact test with Type I error of $5 \%$, will have $86 \%$ power to replicate the difference in the proportion of individuals with NHE reductions. A 2-sided, 2-sample, Wilcoxon-Mann-Whitney test with Type I error of $5 \%$, will have $89 \%$ power to replicate the difference in NHE counts in the prior 6 months.

\section{Discussion}

The study procedures outlined above are intended to perform a SMART efficacy replication trial while working to recruit and retain people with epilepsy in rural and semi-rural communities and to evaluate some of the barriers to self-management particular to rural communities. Rural communities are composed of heterogeneous populations, which have their own needs and vulnerabilities. Many migrant workers and indigenous people live in rural regions, and rural communities have larger proportions of people over the age of 65 and of people with disabilities than urban communities [3]. Many people with epilepsy live in geographically isolated rural communities, and it is important to understand how transportation, access to resources and education, and community attitudes, impact epilepsy care and self-management efficacy. Nationwide there is a shortage of neurologists, but this is especially true in rural communities [37]. It is important to understand how we can best empower people with epilepsy who live in rural communities, and it is possible that self-management may help address some of the gaps in care for rural communities.

Innovative and important aspects of the proposed project include a focus on people with epilepsy who live in under-served and rural communities and use of a lived-experience and provider stakeholder group composed of individuals from under-served and rural communities to inform the use of best practices to maximize outreach/recruitment and retention. In addition, this study method allows a focus on pragmatic epilepsy outcomes relevant to both PLWE and payers/policymakers including seizure occurrence, emergency department visits, hospitalizations and self-harm attempts.

\section{TRIAL STATUS}


We used protocol version 2 from July 2, 2020, and anticipate starting recruitment for the clinical trial in April of 2021, and completing recruitment by the end of 2023.

\section{Abbreviations}

PLWE

People Living with Epilepsy

SMART

Self-management for people with epilepsy and a history of negative health events CWRU

Case Western Reserve University

RCT

Randomized Control Trial

WL

Waitlist

NHE

Negative Health Event

UI

University of lowa

$\mathrm{CAB}$

Community Advisory Board

IRB

Institutional Review Board

REDCap

Research Electronic Data Capture

EHR

Electronic Health Records

PHQ-9

Patient Health Questionnaire 9-question depression scale QOLIE-31

Quality of Life in Epilepsy 31-item short form

ESES

Epilepsy Self-Efficacy Scale

MSPSS

Multidimensional Scale of Perceived Social Support

SSE

Stigma Scale of Epilepsy

WHO

World Health Organization

ILAE 
International League Against Epilepsy

IBE

International Bureau for Epilepsy

\section{Declarations}

Ethics approval and consent to participate: Informed consent will be obtained for all focus group members, peer educators and RCT participants, and all work will be conducted consistent with Institutional Review Board (IRB) approval at the relevant sites. University of lowa's Institutional Review Board (IRB) will serve as the IRB of Record (IRB ID 201910824).

Consent for publication: This paper has not been previously published and is not under consideration by any other journal. All authors consent to publication. We will be willing to provide a model consent form on request.

Availability of data and materials: The authors of this paper at UI and CWRU will have full access to the final trial dataset.

Competing Interests: M.S. has research grants from Otsuka, Alkermes, Nuromate, the International Society of Bipolar Disorders (ISBD), National Institute of Health $(\mathrm{NIH})$, and the Centers for Disease Control and Prevention (CDC). M.S. is a consultant to Alkermes, Otsuka, Janssen, Neurocrine, Bracket, Health Analytics and Frontline Medical Communications and has received publication royalties from Springer Press, Johns Hopkins University Press, Oxford Press, UpToDate. The remaining authors declare that they have no competing interests.

Funding: This publication is a product of a Health Promotion and Disease Prevention Research Center supported by Cooperative Agreement Number 6 U48DP006389 from the Centers for Disease Control and Prevention (CDC). The findings and conclusion in the report are those of the authors and do not necessarily represent the official position of the Centers for Disease Control and Prevention. Support was received from the Institute for Clinical and Translational Science at the University of lowa and the Clinical and Translational Science Collaborative (CTSC) of Cleveland which are both funded by the National Institutes of Health (NIH), National Center for Advancing Translational Science (NCATS), Clinical and Translational Science Award (CTSA) grants, UL1TR002537 (lowa) and UL1TR002548 (Cleveland). The content is solely the responsibility of the authors and do not necessarily represent the official views of the $\mathrm{NIH}$.

Authors' Contributions: GG is one of the prime investigators; she helped refine the protocol and write the manuscript. FB is a co-investigator and statistician who helped to develop outcome measurements and study design. He supervises the data management team made up of the Data Coordinator and the IPC. The data management subcommittee will ensure data integrity, completeness, and accuracy will be verified on an ongoing basis by this committee. $\mathrm{KC}$ is a project manager who assisted with developing study operations. MP helped to improve the plan for recruitment and implementation of the trial. CB is a 
co-investigator and took the lead in qualitative research analysis. MS is one of the prime investigators and led and developed the protocol. All authors read and approved the final manuscript.

Acknowledgements: The authors would like to thank Celeste Weise for her help with reviewing and improving the manuscript.

\section{References}

[1] England MJ, Liverman CT, Schultz AM, Strawbridge LM. Epilepsy across the spectrum: promoting health and understanding. A summary of the Institute of Medicine report. Epilepsy Behav. 2012; 25(2):266-76. doi: 10.1016/j.yebeh.2012.06.016.

[2] Wiebe S, Camfield P, Jette N, et al. Epidemiology of epilepsy: prevalence, impact, comorbidity and disparities. Can J Neurol Sci. 2009;36 Suppl 2:S7-16.

[3] Parker K, Horowitz J, Brown A, Fry R, Cohn D, Igielnik R. What Unites and Divides Urban, Suburban, and Rural Communities. Washington, DC: Pew Research Center;2018.

[4] Carpenter-Song E, Snell-Rood C. The changing context of rural America: a call to examine the impact of social change on mental health and mental health care. Psychiatr Serv. 2017; 68:503-506. doi: 10.1176/appi.ps.201600024

[5] Cosby AG, McDoom-Echebiri MM, James W, Khandekar H, Brown W, Hanna HL. Growth and persistence of place-based mortality in the United States: the rural mortality penalty. Am J Public Health. 2019; 109:155-162. doi: 10.2105/AJPH.2018.304787

[6] Hawley SR, Paschal AM, Ablah E, St Romain T, Liow K, Molgaard CA. Initial perspectives from Midwestern neurologists: epilepsy patients' barriers and motivators for seeking treatment. Epilepsia, 48 (2007), pp. 1920-1925

[7] Szaflarski M. Social determinants of health in epilepsy. Epilepsy Behav. 2014;41:283-289.

[8] Sajatovic M, Jobst BC, Shegog R, et al. The Managing Epilepsy Well Network: Advancing Epilepsy SelfManagement. American Journal of Preventive Medicine. 2017;52(3):S241-S245.

[9] Perzynski AT, Ramsey RK, Colon-Zimmermann K, et al. Barriers and facilitators to epilepsy selfmanagement for patients with physical and psychological co-morbidity. Chronic Illn. 2017;13(3):188203.

[10] Sajatovic M, Colon-Zimmermann K, Kahriman M, et al. A 6-month prospective randomized controlled trial of remotely delivered group format epilepsy self-management versus waitlist control for high-risk people with epilepsy. Epilepsia. 2018;59(9):1684-1695 
[11] Blixen C, Perzynski A, Cage J, et al. Using focus groups to inform the development of stroke recovery and prevention programs for younger African-American (AA) men. Top Stroke Rehabil. 2015;22(3):221230.

[12] Blixen C, Perzynski A, Cage J, et al. Stroke recovery and prevention barriers among young africanamerican men: potential avenues to reduce health disparities. Top Stroke Rehabil. 2014;21(5):432442.

[13] Creswell JW, Qualitative Inquiry \& Research Design: Choosing Among Five Approaches. 2007, Thousand Oaks, CA: Sage.

[14] Denzin NK. The discipline and practice of qualitative research. in The Sage handbook of qualatitive research. N.K. Denzin and Y.S. Lincoln. Editors. Thousand Oaks, CA: Sage; 2005.

[15] Brady TJ, Sniezek J, Ramsey LA. News from the CDC: Scaling up sustainable intervention deliverylessons learned from the CDC arthritis program. Transl Behav Med. 2012;2(1):3-5.

[16] U.S. Department of Agriculture Economic Research Service. Rural-Urban Continuum Codes. 2013. https://www.ers.usda.gov/data-products/rural-urban-continuum-codes.aspx. Accessed 2020.

[17] Blixen C, Perzynski A, Kanuch S, et al. United in purpose: The experience of becoming a peer educator for patients with serious mental illness and diabetes. . Primary Healthcare Research and Development. $2015 ; 16 ; 127-137$

[18] Fraser MW, Richman JM, Galinsky MJ, et al., Intervention research developing social programs. 2009, New York: Oxford University Press.

[19] Miller MD, Paradis CF, Houck PR, et al. Rating chronic medical illness burden in geropsychiatric practice and research: application of the Cumulative Illness Rating Scale. Psychiatry Res. 1992;41(3):237248.

[20] Chaudhry S, Jin L, Meltzer D. Use of a self-report-generated Charlson Comorbidity Index for predicting mortality. Med Care. 2005;43(6):607-615.

[21] Wallace L, North American Primary Care Research G. Patients' health literacy skills: the missing demographic variable in primary care research. Ann Fam Med. 2006;4(1):85-86.

[22] Harris PA, Taylor R, Thielke R, Payne J, Gonzalez N, Conde JG. Research electronic data capture (REDCap)-a metadata-driven methodology and workflow process for providing translational research informatics support. J Biomed Inform. 2009 Apr;42(2):377-81. doi: 10.1016/j.jbi.2008.08.010. Epub 2008 Sep 30. PMID: 18929686; PMCID: PMC2700030.

[23] Kroenke K, Spitzer RL, Williams JB. The PHQ-9: validity of a brief depression severity measure. J Gen Intern Med. 2001;16(9):606-613. 
[24] Ruta D, Garratt A, Abdalla M, et al. The SF 36 health survey questionnaire. A valid measure of health status. BMJ. 1993;307(6901):448-449.

[25] Cramer JA, Arrigo C, Van Hammee G, et al. Comparison between the QOLIE-31 and derived QOLIE-10 in a clinical trial of levetiracetam. Epilepsy Res. 2000;41(1):29-38.

[26] Cramer JA, Perrine K, Devinsky O, Bryant-Comstock L, Meador K, Hermann B. Development and cross cultural translations of a 31 item quality of life in epilepsy inventory. Epilepsia 1998;39:81-8.

[27] Dilorio C, Shafer PO, Letz R, et al. Project EASE: a study to test a psychosocial model of epilepsy medication managment. Epilepsy Behav. 2004;5(6):926-936.

[28] Dilorio C, Hennessy M, Manteuffel B. Epilepsy self-management: a test of a theoretical model. Nurs Res. 1996;45(4):211-217.

[29] Dilorio C, Osborne Shafer P, Letz R, et al. The association of stigma with self-management and perceptions of health care among adults with epilepsy. Epilepsy Behav. 2003;4(3):259-267.

[30] Zimet GD, Dahlem NW, Zimet SG, et al. The multidimensional scale of perceived social support. Journal of Personality Assessment. 1988;52:30-41.

[31] Fernandes PT, Salgado PC, Noronha AL, et al. Stigma Scale of Epilepsy: validation process. Arq Neuropsiquiatr. 2007;65 Suppl 1:35-42.

[32] Fernandes PT, Noronha AL, Sander JW, et al. Stigma scale of epilepsy: the perception of epilepsy stigma in different cities in Brazil. Arq Neuropsiquiatr. 2008;66(3A):471-476.

[33] Curran GM, Bauer M, Mittman B, et al. Effectiveness-implementation hybrid designs: combining elements of clinical effectiveness and implementation research to enhance public health impact. Med Care. 2012;50(3):217-226.

[34] Goldstein LH. Effectiveness of psychological interventions for people with poorly controlled epilepsy. J Neurol Neurosurg Psychiatry. 1997;63(2):137-142.

[35] Tatum WOt, Al-Saadi S, Orth TL. Outpatient case management in low-income epilepsy patients. Epilepsy Res. 2008;82(2-3):156-161.

[36] Oosterhuis A. A psycho-educational approach to epilepsy. Seizure. 1994;3(1):23-24.

[37] Dall TM, Storm MV, Chakrabarti R, Drogan O, Keran CM, Donofrio PD, et al. Supply and demand analysis of the current and future US neurology workforce. Neurology 2013;81:470-8.

\section{Figures}




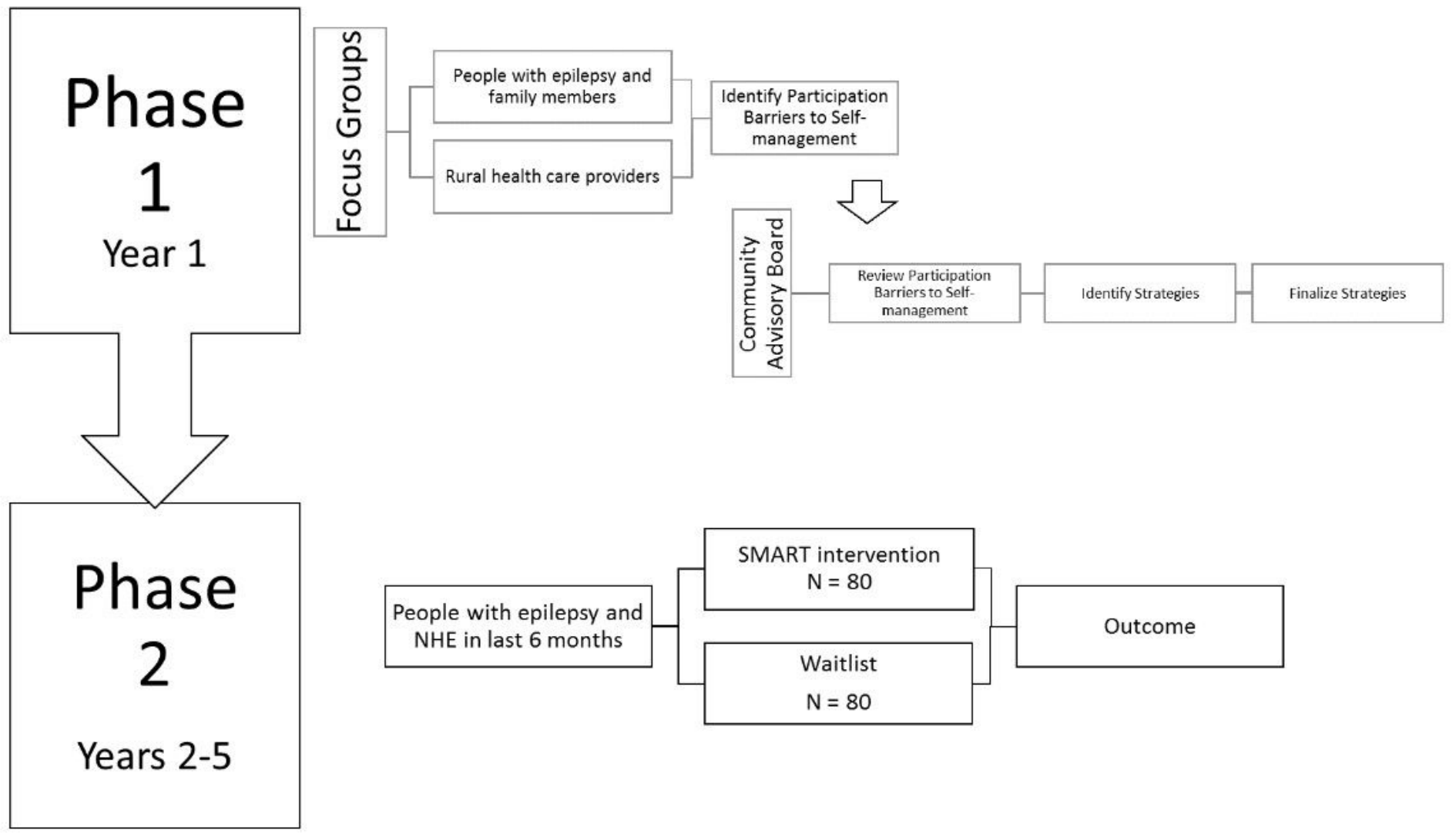

Figure 1

Phase 1 and Phase 2 Study Operationalization NHE = negative health event

\section{Supplementary Files}

This is a list of supplementary files associated with this preprint. Click to download.

- SPIRITChecklist.docx 